ARTICLE

\title{
Simulation for a Real-time Positioning System for Radiotherapy Based on Annihilation Gamma-rays' Detection from a Radiopharmaceutical Concentrated Tumor
}

\author{
Junichi H. KANEKO ${ }^{1 *}$, Eiji TAKADA², Yu HARA ${ }^{1}$, Fumiyuki FUJITA ${ }^{1}$, Toru YAMAGUCHI \\ Naoki KUBO ${ }^{3}$, Masayori ISHIKAWA ${ }^{3}$ and Hiroki SHIRATO ${ }^{3}$ \\ ${ }^{1}$ Graduate School of Engineering, Hokkaido University, N13, W8, Kita-ku, Sapporo,060-8628, Japan. \\ ${ }^{2}$ Toyama National College of Technology, 13, Hongo-machi, Toyama 939-8630, Japan. \\ ${ }^{3}$ Graduate School of Medicine, Hokkaido University, N15, W7, Kita-ku, Sapporo, 060-8638, Japan.
}

\begin{abstract}
To improve radiosurgery for tumors with motion, such as those of lung cancer, a real-time positioning system based on annihilation gamma-ray detection from a radiopharmaceutically concentrated tumor was evaluated using simulations. Several examinations related to the collimator shape were conducted. A pair of wedge-type collimators had good balance in detection efficiency and position sensitivity. As an example of the simulation, a $6 \mathrm{~mm}$ shift of a lung tumor of $5 \mathrm{~cm}$ diameter was detectable with accuracy of 2 sigma using two pairs of detectors, of which the sizes were $20 \times 70 \mathrm{~mm}$.
\end{abstract}

\section{KEYWORDS: radiotherapy, tumor positioning, annihilation gamma-rays, scintillation detectors,}

\section{Introduction}

Since the effectiveness of the tumor radiotherapy was shown, it has been applied to many patients ${ }^{1)}$. For enhancing the therapeutic efficiency, some respiration-gated radiotherapy techniques were developed, which used the information of peritoneum pressure or the movement of the body surface ${ }^{2), 3)}$. However, results demonstrated that the accuracy of those techniques was insufficient. For overcoming the problem, Shirato et al. developed a new technique in which a gold target is set beside the tumor position, which allows the measurement of tumor position ${ }^{4)-6)}$. The system, which enhanced the accuracy of the tumor tracking, was applied to the radiotherapy of the patients.

However, this system presented the disadvantage that the burden for the patient was large because the gold target was set inside their bodies.

To improve the problem, there is a possibility to apply a real-time tumor tracking system based on detection of annihilation gamma rays for this system.

The annihilation gamma rays from the ${ }^{18} \mathrm{~F}$-FDG were used to measure the tumor position. Because the gold target is not necessary using the technique, the burden on the patient can be reduced. In addition, development of a signal processing system to control the new tracking system is described elsewhere ${ }^{7)}$. In this paper, annihilation gamma-ray detection from a radiopharmaceutically concentrated tumor with various types of collimators was evaluated using a semi-analytical simulation.

*Corresponding Author, E-mail:higedon@eng.hokudai.ac.jp (C) 2012 Atomic Energy Society of Japan, All Rights Reserved.

\section{Concept of the tumor positioning system 1. Basic concept}

Figure 1 presents a basic concept of a tumor positioning system evaluated in this study. To identify the tumor position and ambush, the simultaneous detection of the annihilation gamma-rays emitted from a radiopharmaceutical concentrated tumor was evaluated by simulation. Fluorodeoxy glucose (FDG) combined with ${ }^{18} \mathrm{~F}$ was assumed to be used in this simulation. For a lung tumor, 3-8 times accumulation of FDG occurred.

\section{Simulation code and assumption in simulation}

A semi-analytical simulation program was developed in-house; then its accuracy was confirmed using a benchmark experiment with a ${ }^{22} \mathrm{Na}$ radio active source. The simulation code calculates the rate of coincidence of a pair of detectors from a volume source.

In this simulation, a target tumor was determined as lung cancer. It moved by breathing. In the typical case, the tumor position moves more than $10 \mathrm{~mm}$ during exhalation and inhalation. After exhalation, the tumor stays several seconds at the same position. This period presents the opportunity for irradiation. Consequently, this system identifies the tumor as located at the irradiation position or not.

A 5-cm-diameter tumor was assumed to be located in the center axis of the lung. The tumor moved on the center axis. The lung diameter was $12 \mathrm{~cm}$. It was located at the center of a 20-cm-thick water phantom. Densities of the water phantom and the lung were 1 and $0.3 \mathrm{~g} / \mathrm{cm}^{3}$, respectively. Activities of normal tissue and tumor were assumed as 3.7 $\mathrm{kBq} / \mathrm{cc}$ and $18.5 \mathrm{kBq} / \mathrm{cc}$. These values were adopted from typical values for ${ }^{18} \mathrm{~F}-\mathrm{FDG}$ in a lung tumor. Combination of a lead collimator with $5 \mathrm{~cm}$ thickness and a GSO scintillator 


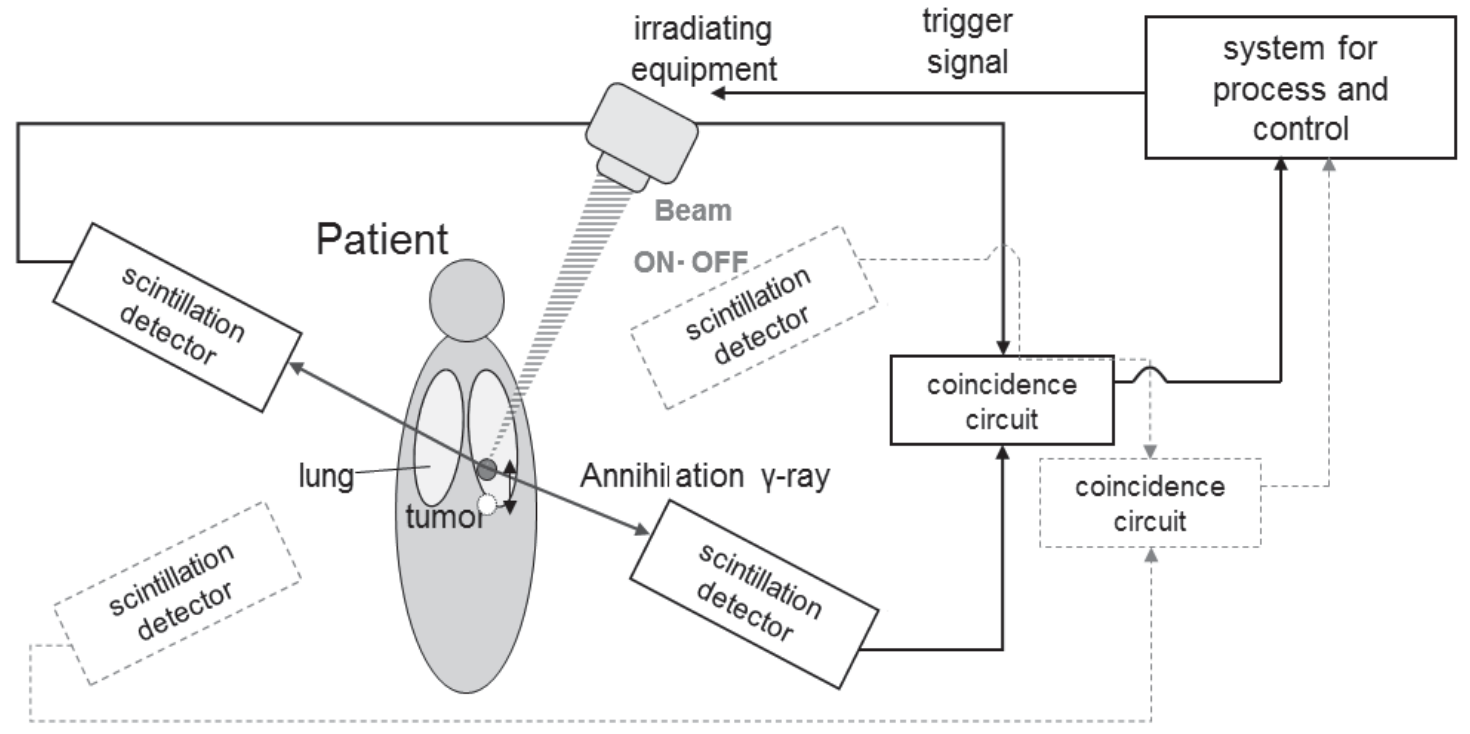

Fig. 1 Basic concept of a tumor positioning system based on annihilation gamma-rays detection.

with $5 \mathrm{~cm}$ thickness were assumed. The distance between oncoming collimators was fixed as $40 \mathrm{~cm}$. The detection efficiency of simultaneous measurement was 0.68 ; this value was obtained in the benchmark experiment using GSO scintillators. In each calculation step, a measurement period of $300 \mathrm{~ms}$ was assumed, in which the tumor remains within the $1 \mathrm{~mm}$ region. The target resolution of the tumor shift was less than $5 \mathrm{~mm}$ in this assumption.

\section{Calculated results}

\section{Consideration on shape of collimators}

At the first step, the collimator shape was investigated. Figure 2 presents dependence of the count rate on the position of a point source for collimators of several shapes. In this calculation, collimators of three types, i.e., a cylindrical type, a circular type and a wedge type was evaluated. For consideration of collimeter shape with high differential of count rate, a crescent type was also considered. In this simulation, the tumor moved on one axis. Therefore, the wedge type collimator had a sharp change in the count rate.

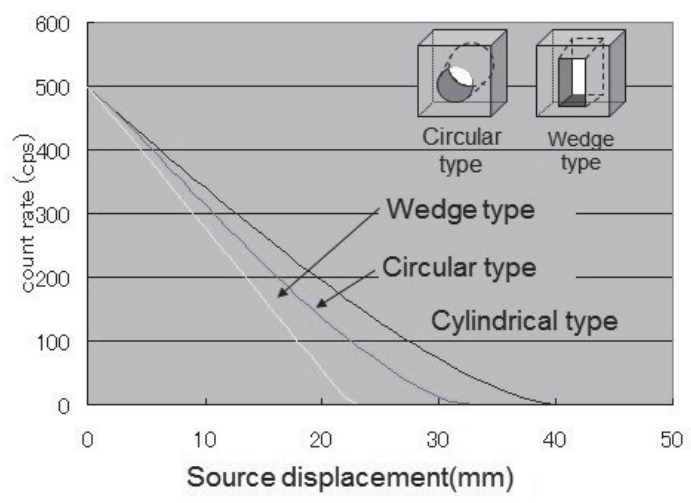

Fig. 2 Dependence of count rate on position of a point source to collimators.
For further investigation of the collimator shape, the distribution of activity in the tumor from the line of sight, consisting of two detectors, was considered. It is shown in Figure 3a). The differential of count rate with a shift of the tumor in $5 \mathrm{~mm}$ is shown in Figure 3b). This result showed that the crescent shape collimator had very high sensitivity for the shift of this tumor. However, more than 30 pairs of detectors were necessary to obtain a sufficient count in 300 ms.
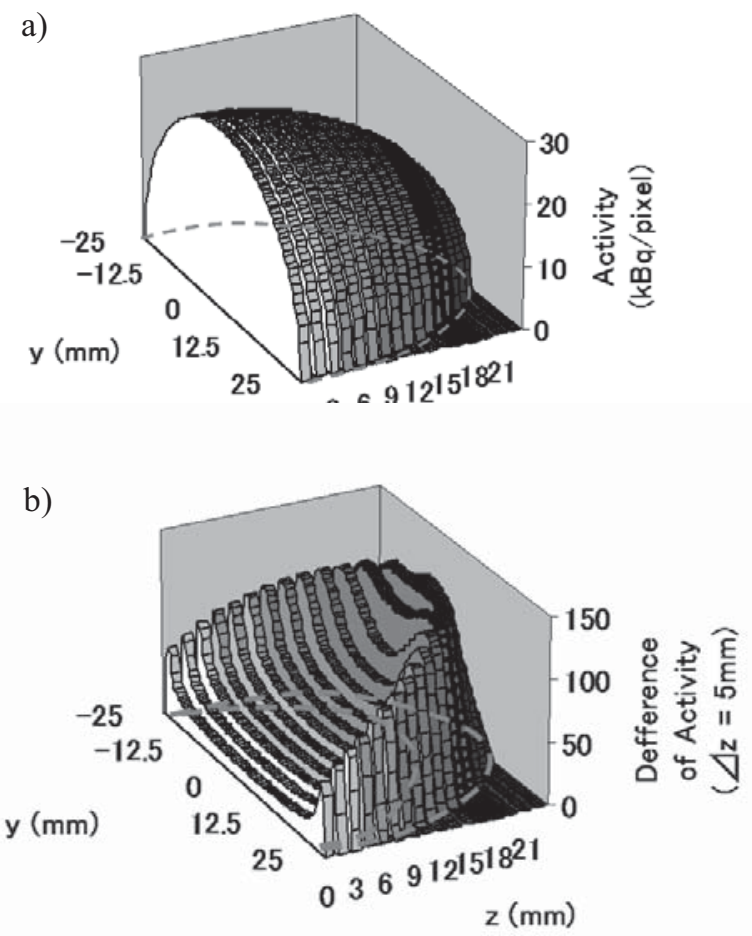

Fig. 3 Distribution of a) activity, b) differential of count rate with a shift of the tumor in $5 \mathrm{~mm}$ from a line of sight. 
From these considerations, a rectangular window, i.e., wedge type collimator, was found to be a superior and realistic choice to obtain high sensitivity for the shift of tumor and sufficient detection efficiency.

\section{Consideration on detector width}

For practical system design, two pairs of detectors were useful for irradiation, and four pairs of detectors were regarded as an upper limit. Then the detector window area was fixed at $14 \mathrm{~cm}^{2}$. This area was adopted from easy optical connection with a photomultiplier tube of $51 \mathrm{~mm}$ diameter. Figure 4 presents dependence of the change of count rate with $5 \mathrm{~mm}$ shift of tumor on several detector widths.

In addition, the dependence of ratio of the differential of count rate for error on detector width is shown in Figure 5. Here $\mathrm{Dz}$ was intended as the shift of the tumor, i.e. $5 \mathrm{~mm}$. Based on this result, a $20-\mathrm{mm}$ detector width was inferred as the best choice for the calculated assumption.

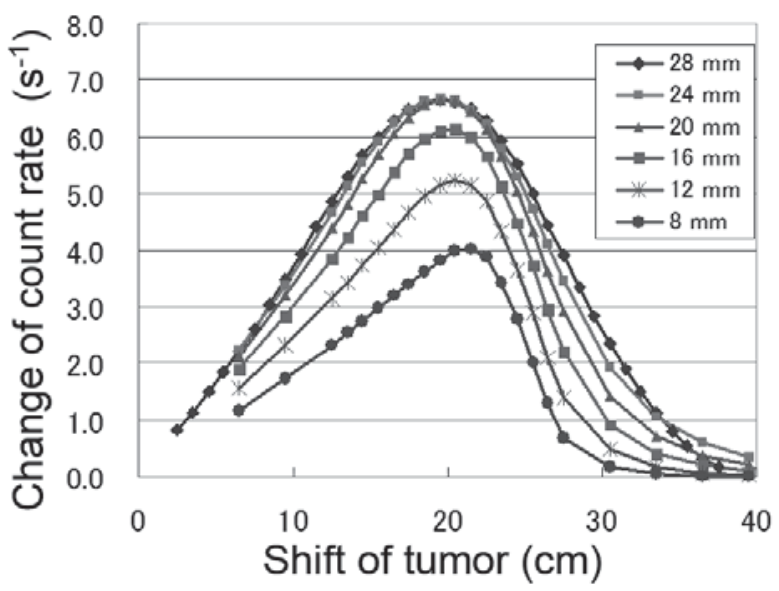

Fig. 4 Dependence of change of count rate on detector width. Here, $\mathrm{D}_{\mathrm{z}}$ was $5 \mathrm{~mm}$.

Figure 5 presents an example of change of count rate $(\Delta \mathrm{N})$ for error on detector width $\left(\mathrm{N}_{\text {error }}\right)$ on the tumor position: two pairs of detector with wedge-type collimators. The detector width was $20 \mathrm{~mm}$. The detector center was shifted $18 \mathrm{~mm}$ on the $\mathrm{Z}$ axis. In this case, detection of the tumor shift in $6 \mathrm{~mm}$ with accuracy in $2 \sigma$ was achieved.

The required sensitivity of $5 \mathrm{~mm}$ for the shift of tumor will be possible by further optimization of a collimator shape and/or the increase of detector pairs.

\section{Conclusions}

The concept model of tumor tracking system using annihilation gammma-ray detection was evaluated by semi-analytical simulation.

Three types of collimators, circular, cylindrical and wedge type, were tested. A pair of wedge-type collimators with

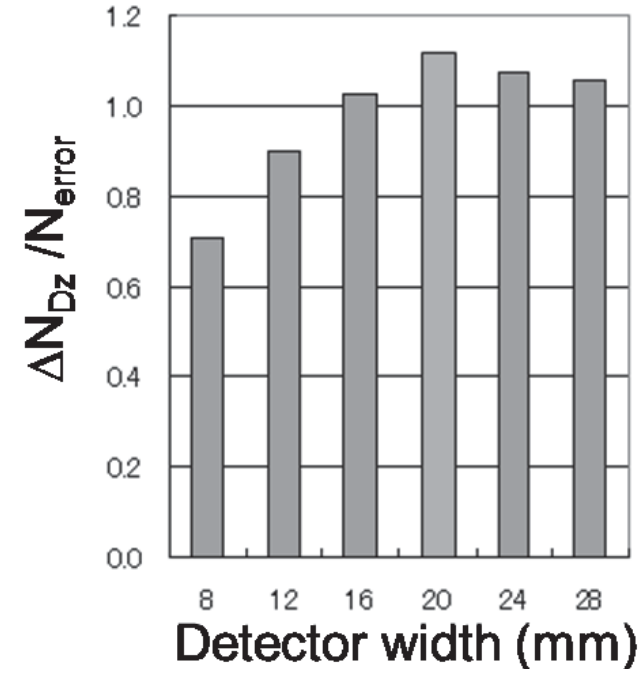

Fig. 5 Dependence of ratio of change of count rate for error on detector width.

$20 \mathrm{~mm}$ width had good balance in detection efficiency and position sensitivity.

Further evaluation for real tumors are required in the next step.

\section{Acknowledgment}

This study was financially supported by KAKENHI (Grant No.17016002).

\section{References}

1) H. Shirato, T. Isu, S. Matsumura, et al., "Daily intermittent multi-portal therapy followed by stereotactic boost (DIMTSB) for treatment of small intracranial lesions: Technical aspects and preliminary results", J. Jpn. Soc. Ther. Radiol. Oncol, 4,149 $-162(1992)$.

2) K. Randall. H. Ten, J. M. Balter, et al., "Potential benefits of eliminating planning target volume expansions for patient breathing in the treatment of liver tumors", Int. J. Radiat. Oncol. Biol. Phys., 38, 613-617(1997).

3) H. D. Kubo, B. C. Hill, "Respiration-gated radiotherapy treatment: a technical study", Phys. Med. Biol., 41, 83-91(1996).

4) H. Shirato, S. Shimizu, et al. "Four-dimensional treatment planning and fluoroscopic real-time tumor tracking radiotherapy for moving tumor", Int. J. Radiat. Oncol. Biol. Phys., 48, 435-442(2000).

5) H. Shirato, S. Shimizu, et al., "Physical aspects of a real-time tumor-tracking system for gated radiotherapy”, In.t J. Radiat. Oncol. Biol. Phys., 48, 1187-1195 (2000).

6) S. Shimizu, H. Shirato, et al., "Detection of lung tumor movement in real-time tumor-tracking radiotherapy", Int. J. Radiat. Oncol. Biol. Phys., 51, 2, 304-310 (2001).

7) E. Takada, J. H. Kaneko, et al., "Development of a Signal Processing system to Control the Tumor Positioning System Based on the Annihilation Gamma-rays Measured by Several Pairs of Scintillation Detectors", submitted to Progress in Nuclear Science and Technology. 